\title{
Determining the Relative Configuration of Propargyl Cyclopro- panes by Co-Crystallization
}

\author{
Felix Krupp ${ }^{a}$ \\ Marie-Idrissa Picher ${ }^{a}$ \\ Wolfgang Frey ${ }^{a}$ \\ Bernd Plietker ${ }^{b}$ \\ Clemens Richert*a \\ a Institut für Organische Chemie, Universität Stuttgart, \\ 70569 Stuttgart, Germany \\ lehrstuhl-2@oc.uni-stuttgart.de

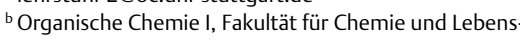 \\ mittelchemie, Technische Universität Dresden, 01069 \\ Dresden, Germany
}
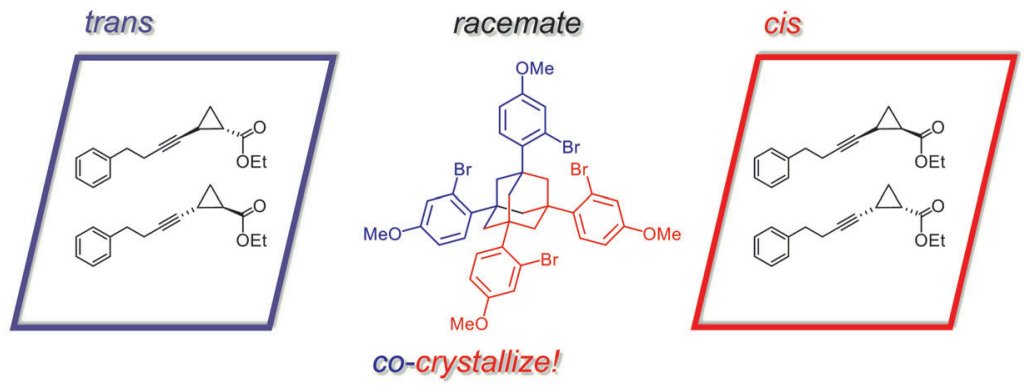

co-crystallize!
Received: 18.09.2020

Accepted after revision: 20.10.2020

Published online: 20.10 .2020

DOI: 10.1055/a-1293-9867; Art ID: st-2020-b0511-I

License terms: cc)

(c) 2021. The Author(s). This is an open access article published by Thieme under the terms of the Creative Commons Attribution-NonDerivative-NonCommercial-License, permitting copying and reproduction so long as the original work is given appropriate credit. Contents may not be used for commercial purposes or adapted, remixed, transformed or built upon. (https://creativecommons.org/licenses/by-nc-nd/4.0/)

Abstract Determining the diastereoselectivity of new synthetic molecules can be a challenge when NMR methods fail and the compounds are difficult to crystallize. Encapsulating organic crystals can be used to overcome this challenge. Here we show that the diastereomeric configuration of racemic mixtures of propargyl cyclopropanes can be determined by co-crystallization with 1,3,5,7-tetrakis(2-bromo-4-methoxyphenyl)adamantane and X-ray crystallography. Three crystal structures are reported that unambiguously identify the products of Fe-catalyzed cyclopropanations as cis- or trans-isomers. These findings expand the scope of co-crystallization with tetraaryladamantanes as a method to determine the stereochemical configuration of organic molecules that are difficult to crystallize by themselves.

Key words stereochemistry, crystallization, diastereomers, structure elucidation, X-ray crystallography

Many reactions in organic synthesis produce more than one stereoisomer. Distinguishing between diastereomers produced in synthetic transformations is usually achieved by NMR spectroscopy, as two diastereomers give different NMR ${ }^{1} \mathrm{H}$ and ${ }^{13} \mathrm{C}$ signals. However, the differences in chemical shift and coupling constants between the two diastereomers may be too small to allow for unambiguous detection, and peak overlap may prevent unambiguous assignment when the complexity and the size of a molecule increases. Some sets of diastereomers, such as those of fusarins G1/G2 and $\mathrm{G} 3 / \mathrm{G} 4$, isolated from Mucor fungi, even show nearidentical ${ }^{1} \mathrm{H}$ and ${ }^{13} \mathrm{C}$ signals and similar $J$-coupling patterns, rendering assignment of their configurations extremely difficult. ${ }^{1}$ Although those issues can be overcome by detailed analysis using two-dimensional NMR methods, ${ }^{2,3}$ determi- nation of the configuration and structure of a molecule with NMR spectroscopy is more error-prone than X-ray crystallographic determination. Unfortunately, the latter method usually requires single crystals of sufficient size and quality, which can be difficult to obtain for a given set of analytes.

One way to obtain single crystals for difficult-to-crystallize compounds is co-crystallization with a 'chaperone' that provides a crystalline scaffold. However, a dearth of reliable chaperones for small organic molecules has made it difficult to employ this concept in organic synthesis. ${ }^{4}$ Recently, we reported a one-step thermal crystallization method for determining the absolute configuration of small molecules by co-crystallization. ${ }^{5}$ This method uses tetraaryladamantane tetra- or octaethers as chaperones. Tetraaryladamantanes are known to form crystalline inclusion complexes with a wide range of small molecules, ${ }^{6-9}$ and the resulting solids have been referred to as 'encapsulating organic crystals' (EnOCs). ${ }^{10}$ Thermal co-crystallization is rapid and does not require screening for suitable solvents. It produced well-diffracting co-crystals for the majority of over 50 liquid compounds tested. ${ }^{5}$

This prompted us to ask whether co-crystallization might also be used to determine relative configurations of compounds prepared in nonenantioselective synthetic processes. Racemic mixtures are usually more difficult to crystallize than pure compounds of a single stereochemical configuration. Co-crystallizing such enantiomeric mixtures was therefore considered even more demanding than cocrystallizing chiral compounds. The iron-catalyzed cyclopropanation of 1,3-enynes (Scheme 1 ) provided an opportunity to put our method to the test. The underlying methodology, published recently, ${ }^{11}$ produces cis- and trans-propargyl cyclopropane diastereomers as racemic mixtures.

Whereas the determination of the relative configuration of substituted cyclopropanes can often be achieved on the basis of the vicinal coupling constants of cyclopropyl protons $\left({ }^{3} J_{\text {cis }}>{ }^{3} J_{\text {trans }}\right.$; i.e. ${ }^{3} J_{\text {cis }}=7-10 \mathrm{~Hz}$ and $\left.{ }^{3} J_{\text {trans }}=2-6 \mathrm{~Hz}\right),{ }^{12-14}$ 


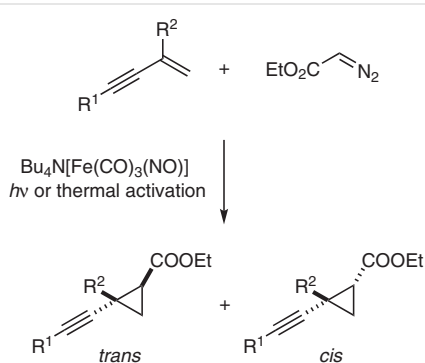

Scheme 1 Iron-catalyzed cyclopropanation of 1,3-enynes under photochemical or thermal activation. $\mathrm{R}^{1}=\mathrm{Ar}$, Alk, $\mathrm{C}(\mathrm{O}) \mathrm{R}, \mathrm{CH}_{2} \mathrm{OR}, \mathrm{CH}_{2} \mathrm{NR}$; $\mathrm{R}^{2}=\mathrm{H}$, Alk.

the assignment becomes less straightforward when coupling constants are in the range of $6-7 \mathrm{~Hz}$, or when the signals of the cyclopropyl protons overlap with the signals of aliphatic protons. Since in-depth 2D NMR analysis requires long acquisition times and detailed data analysis, fraught with potential pitfalls, and because only a few of the newly synthesized cyclopropane derivatives crystallized by themselves, we were hoping that the novel co-crystallization process with tetraaryladamantanes would provide rapid and unambiguous answers. Here, we report four co-crystal structures for products of the cyclopropanation reaction mentioned above, together with the relative configuration of the products.

Figure 1 shows the structures of the propargyl cyclopropanes analytes selected for the co-crystallization study, together with that of 1,3,5,7-tetrakis(2-bromo-4-methoxyphenyl)adamantane (TBro), which was chosen as crystallization chaperone, as it had previously proven suitable for forming crystalline inclusion compounds with analytes of molecular weights of up to about $250 \mathrm{~g} / \mathrm{mol} .{ }^{5}$ This tetrabromide is also a good co-crystallization agent for lipophilic compounds that might not interact strongly enough with octaether chaperones to form crystalline inclusion complexes. ${ }^{5,6}$

All conventional crystallization experiments for racemic cis- and trans-cyclopropanes $\mathbf{1}$ and $\mathbf{2}$ had failed when we began our experiments, indicating that the analytes have little tendency to crystallize by themselves. Co-crystallization was induced by treating $30 \mu \mathrm{L}$ of HPLC-purified, racemic 1 with $5 \mathrm{mg}$ of $\mathbf{T B r o}$, heating to $70{ }^{\circ} \mathrm{C}$ in a glass vial placed on the surface of a heater/stirrer until a clear solution formed, and allowing crystallization to occur by switching off the hotplate. A crystal suitable for X-ray crystallography was picked on the next day and proved suitable to elucidate the relative configuration. This, together with analogous experiments with the other three analytes, gave the results shown in Figure 2 and in Table 1, entries 1-4. Further details of the crystallization and the X-ray crystal structures are provided in the Supporting Information. The coordinates have been deposited with the Cambridge Crystallographic Data Centre. ${ }^{15}$
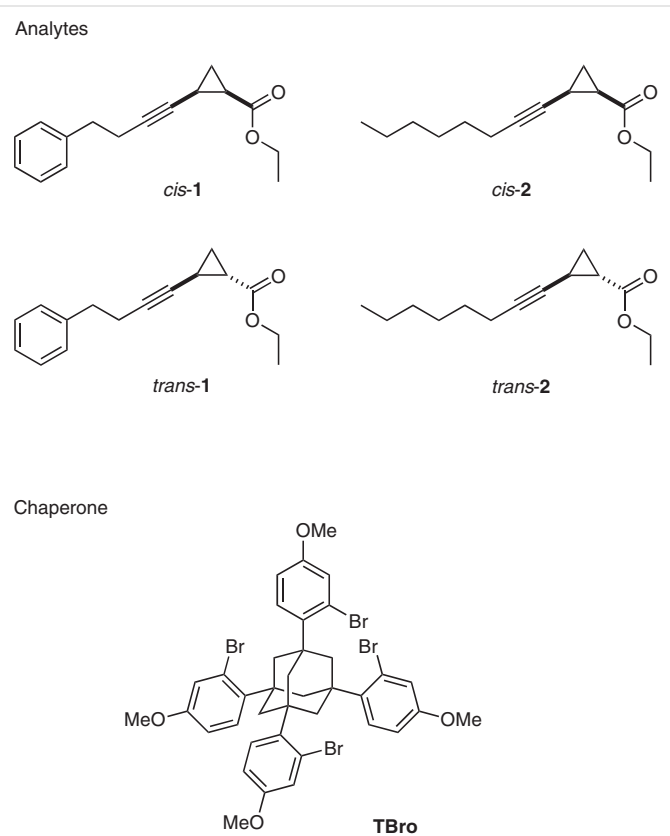

Figure 1 Cyclopropane analytes, that were obtained as racemic mixtures, and the crystallization chaperone used for co-crystallization

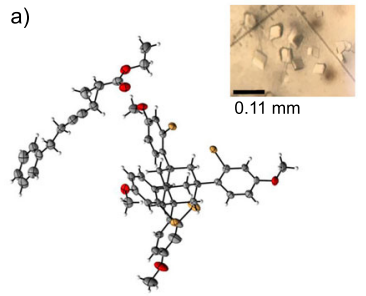

TBro / trans-1, monoclinic, P2(1)/n

c)

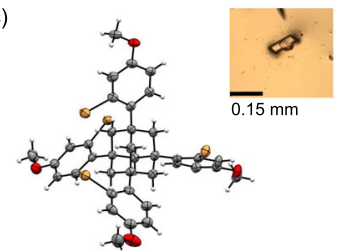

TBro alone crystallized from cis-2, monoclinic, $\mathrm{C} 2 / \mathrm{c}$
TBro / cis-1, trigonal, R-3

d)

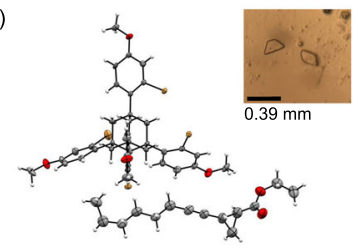

TBro / trans-2, triclinic, $\mathrm{P}-1$

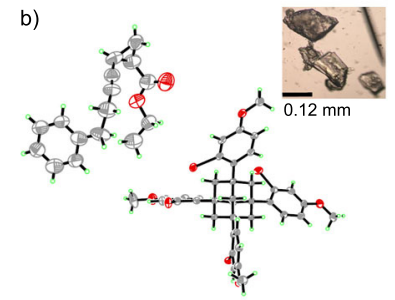

Figure 2 Details of crystal structures obtained in thermal crystallization runs together with photographs of crystals, including black length bars in the lower left-hand corner of each photograph. (a) TBro co-crystallized with trans-1, (b) TBro co-crystallized with cis-1, (c) TBro by itself, as obtained from an attempt to co-crystallize it with cis-2, and (d) TBro co-crystallized with trans-2. The components of the crystallization mixture and the crystal system are given below each structural drawing (ORTEP plots on $50 \%$ probability level). 
Table 1 Quantities Used for Co-Crystallization Experiments and Results of X-Ray Crystallographic Analyses

\begin{tabular}{lllll}
\hline $\begin{array}{l}\text { TBro } \\
{[\mathrm{mg}]}\end{array}$ & $\begin{array}{l}\text { Analyte } \\
(\text { Vol }[\mu \mathrm{L}])\end{array}$ & Crystal system & $\begin{array}{l}\text { Space } \\
\text { group }\end{array}$ & $R 1$ \\
\hline 5 & cis-1 $(30)$ & trigonal & $R 3$ & 0.0296 \\
1 & trans-1 (50) & monoclinic & $P 2_{1} / n$ & 0.0575 \\
1 & cis-2 (30) & (monoclinic) & $C 2 / c$ & 0.0499 \\
1 & trans-2 (30) & triclinic & $P 1$ & 0.0602 \\
1 & $1: 4$ cis-1/trans-1 (50) & monoclinic & $P 2_{1} / n$ & 0.0657 \\
\hline
\end{tabular}

${ }^{a}$ Crystallization of TBro alone; no inclusion of analyte.

b Only racemic trans-1 was observed in the co-crystal.

Three of the four cyclopropanes formed crystalline inclusion complexes with TBro, whereas attempts to co-crystallize cis-2 with the tetraaryladamantane led to crystals of the chaperone alone. In all three cases, racemic co-crystal structures were obtained, meaning that equimolar amounts of one the two enantiomers were encapsulated upon crystallization. The crystallographic data allowed the unambiguous assignment of the configuration of cis- and trans-1, as well as that of trans-2 (Figure 2).

Figure 3 shows the crystal packing for trans- $\mathbf{1}$ as a representative case. There are two molecules of the analyte in the asymmetric cell. One of them (lower right-hand corner of Figure $3 \mathrm{a}$ ) is well ordered and was used to determine the relative configuration. The other molecule, on the left-hand side of the same figure, is partially disordered. Together, the analytes form layers interleaved between well-ordered TBro molecules (Figure 3b). This arrangement apparently leads to a reasonable level of space filing, a requirement for obtaining well-resolved diffraction patterns for analytes. Three different crystal systems were adopted by TBro in our crystals: trigonal, monoclinic, and triclinic.

The ability to find diverse crystalline arrangements, including arrangements where an imperfect shape complementarity leads to local disorder (but a sufficient number of analyte molecules are well ordered for structure elucidation) helps to explain why molecules structurally different from each other (and the many other guest molecules studied earlier $)^{5-9}$ can be encapsulated in EnOCs by using chaperones like TBro. As in our previous study on determining absolute configuration by co-crystallization, ${ }^{5}$ the current approach does not require screening for solvents or special crystallization conditions, and it produced final results in less than 48 hours. We have yet to determine whether crystallization can be performed without heating, as in the 'spectroscopy cum crystallization' (SCC) method reported earlier. ${ }^{5}$

The results shown in Table 1, entries 1-4 were obtained with one racemic mixture (cis or trans), as isolated after chromatographic purification. As a first foray into structure elucidation for more complex mixtures, as often obtained in synthetic transformations, we then used a 1:4 mixture of a)

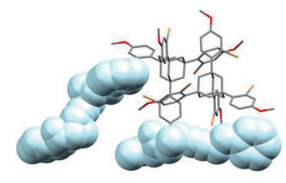

b)

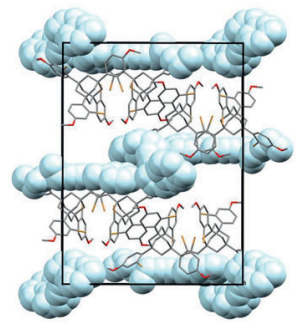

Figure 3 Details of the co-crystal structure of TBro and trans-1. (a) Asymmetric unit containing one well-ordered molecule and one partially disordered molecule of the analyte; (b) crystal packing of chaperone and analyte molecules in the crystal lattice. The TBro molecules are shown as stick representations, the molecules of trans- $\mathbf{1}$ are spacefilling models in light blue. Hydrogen atoms are omitted for clarity.

the cis- and trans-racemates from the synthetic work, without HPLC separation. When this mixture containing four stereoisomers in total was employed in our co-crystallization process under the conditions mentioned above, a racemic crystal structure containing the dominant diastereomer (trans-1) was obtained (Table 1, entry 5 ). The process of structure elucidation took less than two days in total. The structure obtained (Figure 4) again showed one set of wellordered analyte molecules suitable for determining the relative configuration with accuracy.

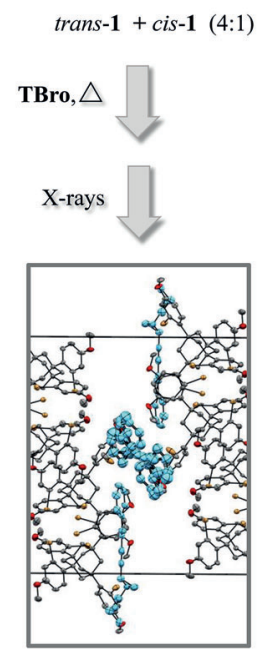

Figure 4 Co-crystallization starting from a 4:1 mixture of trans-1 and cis-1, with TBro as crystallization chaperone. The lower part of the figure shows the position of analyte molecules in the unit cell in light blue, with $\mathrm{H}$ atoms omitted for clarity. Two well-ordered molecules are in an extended conformation, positioned vertically; two disordered analytes are in the center of the detail of the crystal structure shown. 
In summary, co-crystallization of racemic mixtures of propargyl cyclopropanes with TBro as crystallization chaperone showed that EnOCs are useful for solving stereochemical issues beyond pure enantiomers. This broadens the scope of co-crystallization with molecular chaperones as a method for the structure elucidation of small molecules that are difficult to crystallize by themselves. The procedure is rapid, often producing a result in a single thermal crystallization run, and requires smaller amounts of analytes than typical conventional crystallization techniques. Given the importance of stereochemistry in organic chemistry, the reported technique has the potential to accelerate studies in method development and catalysis, and might also be helpful in elucidating the structure of natural products.

\section{Funding Information}

This work was supported by DFG grant RI 1063/17-1.

\section{Acknowledgment}

The authors thank D. Göhringer and O. Bernhard for technical assistance.

\section{Supporting Information}

Supporting information for this article is available online at https://doi.org/10.1055/a-1293-9867.

\section{References and Notes}

(1) Wang, C.-X.; Chen, G.-D.; Feng, C.-C.; He, R.-R.; Qin, S.-Y.; Hu, D.; Chen, H.-R.; Liu, X.-Z.; Yao, X.-S.; Gao, H. Chem. Commun. 2016, $52,1250$.

(2) Bross-Walch, N.; Kühn, T.; Moskau, D.; Zerbe, O. Chem. Biodivers. 2005, 2, 147.

(3) Bifulco, G.; Dambruoso, P.; Gomez-Paloma, L.; Riccio, R. Chem. Rev. 2007, 107, 3744.

(4) Bhatt, M. B.; Desiraju, G. R. CrystEngComm 2008, 10, 1747.

(5) Krupp, F.; Frey, W.; Richert, C. Angew. Chem. 2020, 132, 16009; Angew. Chem. Int. Ed. 2020, 59, 15875.

(6) Schwenger, A.; Frey, W.; Richert, C. Chem. Eur. J. 2015, 21, 8781.

(7) Alexandre, P. E.; Schwenger, A.; Frey, W.; Richert, C. Chem. Eur.J. 2017, 23, 9018.

(8) Schwenger, A.; Frey, W.; Richert, C. Angew. Chem. Int. Ed. 2016, 55,13706

(9) Krupp, F.; He, S.; Frey, W.; Richert, C. Synlett 2018, 29, 1707.

(10) Richert, C.; Krupp, F. Synlett 2017, 28, 1763.

(11) Picher, M.-I.; Plietker, B. Org. Lett. 2020, 22, 340.

(12) The Chemistry of the Cyclopropyl Group, Vols. 1 and 2; Rappoport, Z., Ed.; Wiley: Chichester, 1987.

(13) Kretschik, O.; Nieger, M.; Dötz, K. H. Chem. Ber. 1995, 128, 987.

(14) Solladié-Cavallo, A.; Isarno, T. Tetrahedron Lett. 1999, 40, 1579.

(15) CCDC 2032133, 2032113, 2032120, 2032115, and 2032116 contain the supplementary crystallographic data for, respectively, TBro with encapsulated cis-1, trans-1, trans-2, and trans$\mathbf{1}$ (crystallized from a 4:1 mixture of trans- and cis-1), and for TBro without inclusion of the analyte (crystallized from cis-2). The data can be obtained free of charge from The Cambridge Crystallographic Data Centre via www.ccdc.cam.ac.uk/getstructures. 